\title{
Effects of Semantic Priming on Visual Encoding of Pictures
}

\author{
Mark Tippens Reinitz \\ Southeastern Louisiana University \\ Eve Wright and Geoffrey R. Loftus \\ University of Washington
}

\begin{abstract}
We investigated the effects of semantic priming on initial encoding of briefly presented pictures of objects and scenes. Pictures in four experiments were presented for varying durations and were followed immediately by a mask. In Experiments 1 and 2, pictures of simple objects were either preceded or not preceded by the object's category name (e.g., dog). In Experiment 1 we measured immediate object identification; in Experiment 2 we measured delayed old/new recognition in which targets and distractors were from the same categories. In Experiment 3 naturalistic scenes were either preceded or not preceded by the scene's category name (e.g., supermarket). We measured delayed recognition in which targets and distractors were described by the same category names. In Experiments 1-3, performance was better for primed than for unprimed pictures. Experiment 4 was similar to Experiment 2 in that we measured delayed recognition for simple objects. As in Experiments 1-3, a prime that preceded the object improved subsequent memory performance for the object. However, a prime that followed the object did not affect subsequent performance. Together, these results imply that priming leads to more efficient information acquisition. We offer a picture-processing model that accounts for these results. The model's central assumption is that knowledge of a picture's category (gist) increases the rate at which visual information is acquired from the picture.
\end{abstract}

It is well known that preceding a target picture by a semantically related priming stimulus can speed its identification. The typical explanation is that primes influence relatively late cognitive processes-that is, processes for which input is perceptual information acquired from the target picture. For instance, primes might reduce the amount of stimulus information required to gain access to entries in lexical memory or meaning representations in semantic memory (e.g., Ratcliff \& McKoon, 1988).

However, primes may also influence early perceptual processing; that is, they may affect acquisition of initial, perceptual

The research was supported by a grant from the National Institute of Mental Health to Geoffrey R. Loftus. Experiment 1 is part of a doctoral dissertation submitted to the University of Washington by Mark Tippens Reinitz and was presented by Reinitz and Loftus at the 1986 Psychonomic Society meeting in New Orleans. Experiments 2-4 were presented by Reinitz, Eve Wright, and Loftus at the 1988 Psychonomic Society meetings in Chicago.

Our heartfelt thanks go to Rose Ahern, Janine Duncan, Paul Gerhig, and Susan Pierce for running massive numbers of subjects and for their insights, ideas, criticisms, and comments during all phases of the research. A large number of people provided helpful comments on earlier versions of the manuscript, including Tom Carr, Buz Hunt, Beth Loftus, Steven Lupker, Michael Masson, John Miyamoto, John Palmer, Jonathan Schooler, Gay Snodgrass, George Wolford, and an anonymous reviewer. Mark Tippens Reinitz thanks Southeastern Louisiana University for providing release time to write the article.

Correspondence concerning this article should be addressed to Mark Tippens Reinitz, Department of Psychology, Box 831, Southeastern Louisiana University, Hammond, Louisiana 70402. information from the picture. By perceptual information we refer to information that can be acquired only from the physical stimulus or from the icon that follows it (see Loftus, Hanna, \& Lester, 1988; Loftus \& Hogden, 1988). The primary purpose of our research was to investigate this hypothesis by using both naturalistic scenes and simple objects as stimuli. In anticipation of our results, we found that primes do influence information acquisition. Accordingly, a second purpose of our experiments was to begin to determine the nature of this influence.

\section{Contextual Effects on Visual Processing}

Priming is a form of context effect. Many studies have demonstrated context effects on visual processing of both scenes and objects. For instance, the gist of a scene is usually acquired during the first eye fixation on the scene (Intraub, 1981; Loftus, Nelson, \& Kallman, 1983), and gist influences subsequent scanning of the scene (Biederman, Glass, \& Stacy, 1973; Loftus \& Mackworth, 1978). When there is no inherent gist to a picture, visual search times are long (Biederman et al., 1973). Preceding pictures of objects by related picture or word primes speeds both identification and naming (Carr, McCauley, Sperber, \& Parmalee, 1982; Durso \& Johnson, 1979; Irwin \& Lupker, 1983; Sperber, McCauley, Ragain, \& Weil, 1979). Last, subjects are more accurate at identifying primed than unprimed pictures (Palmer, 1975).

Together, these studies indicate that semantic context plays an important role in overall visual processing of pictures. Does this role include any effect on initial information acquisition? The evidence bearing on this question is scanty and 
indirect. In the following sections, we discuss what evidence there is; first, however, we discuss the methodological biases that have resulted in the scantiness of the evidence to begin with.

\section{Methodological Biases}

Most priming studies have not been specifically designed to investigate information acquisition. This has led to three methodological biases. First, most priming experiments are designed to investigate issues concerning long-term memory representation. Second, in few priming experiments have pictures been used as targets. Third, in almost all priming experiments, researchers have measured response latency, which is not ideal for investigating information acquisition. We discuss these three biases in turn.

\section{Priming as a Tool to Investigate Long-Term Memory}

Priming experiments are usually designed to investigate issues such as flow of activation in semantic memory (e.g., Burke, White, \& Diaz, 1987; Ratcliff, Hockley, \& McKoon, 1986; R. E. Warren, 1977), internal representations of sentences and text (e.g., McKoon \& Ratcliff, 1980; Ratcliff \& McKoon, 1978), shared representational structures for words and pictures (e.g., Durso \& Johnson, 1979; Irwin \& Lupker, 1983; Potter, 1979), lexical memory in bilingual speakers (e.g., Kroll \& Curley, 1986; Smith, 1986), semantic/episodic memory differences (e.g., Neely \& Durgunoglu, 1985; Tulving, Schacter, \& Stark, 1982), and retrieval from long-term memory (e.g., Anderson, 1974; Neely, 1977). This focus on long-term memory has naturally led researchers to design paradigms that are optimal for investigating memory effects but suboptimal for investigating perceptual effects. Consider, for example, the well-known priming paradigm invented by Ratcliff and McKoon (1978; McKoon \& Ratcliff, 1980) to study memory representations for sentences. In this paradigm a subject learns sentences during a study phase. Primes are presented during a subsequent verification stage. The typical finding is that words that occurred within the same proposition in a sentence prime one another more than do words from different propositions. For our purposes it is important to note that such a paradigm cannot be used to investigate priming effects on perception because the sentences are not primed at the time when they are perceived. Many other priming paradigms involve presenting primes during a memory test rather than during stimulus encoding (Anderson, 1974; Neely \& Durgunoglu, 1985; Neely, Schmidt, \& Roediger, 1983; Tulving et al., 1982; Warrington \& Weiskrantz, 1968). Such experiments are irrelevant to the issue of priming effects on perception because retrieval, rather than encoding, is primed. It is therefore not surprising that Ratcliff and McKoon (1988) recently proposed a general model in which they attributed priming effects to retrieval processes.

\section{Infrequent Use of Picture Targets}

In most priming studies, researchers use word or letter targets. There exists substantial evidence that there are differ- ences between picture and word processing (Loftus, 1982; Potter \& Faulconer, 1975) and different effects of priming on picture and word targets (Carr et al., 1982; Sperber et al., 1979; C. Warren \& Morton, 1982). Studies in which alphanumeric targets are used are therefore of questionable relevance to issues of picture perception. Those researchers who have used picture targets have generally not addressed picture perception per se. Rather, most have factorially combined picture and word primes with picture and word targets in an attempt to show a common underlying semantic representation for pictures and words (e.g., Carr et al., 1982; Durso \& Johnson, 1979; Kroll \& Potter, 1984; Sperber et al., 1979). Others have presented words and pictures simultaneously in order to demonstrate Stroop-type interference (Lupker, 1979; Lupker \& Katz, 1982). Again, the purpose of the experiments was to show that words and pictures enable one to gain access to a common semantic store, rather than to investigate picture perception per se.

\section{Problems Associated With Response Latency}

In almost all priming studies, researchers use response latency as the dependent measure. The time to respond to a primed target reflects the time to perform a large number of perceptual and cognitive processes, as well as to make a motor response. Thus a change in response time across priming conditions may reflect a change in perceptual processing time, a change in cognitive processing time, or both. Any conclusions about the specific effect of primes must be indirect.

\section{Evidence for Priming Effects on Picture Perception}

Despite these biases, some experiments in the literature do provide indirect evidence for the existence of priming effects on visual information acquisition.

Meyer, Schvaneveldt, and Ruddy (1975) presented primes just before targets to study priming in lexical decision. They showed that the effects of stimulus degradation interact with those of semantic relatedness, and they argued that both manipulations affect perceptual encoding of word targets. Sperber et al. (1979) independently varied degree of target degradation and degree of prime-target relatedness for picture targets; again, the finding was that the two variables interact. Sperber et al. concluded that priming affects a relatively early stage of picture encoding.

C. Warren and Morton (1982) also provided evidence for a role of priming in acquisition of visual information. During a pretraining phase, subjects viewed a series of words and pictures of objects. Recognition thresholds were determined in a subsequent test phase 45 min later. Recognition performance for new pictures was not facilitated by prior viewing of the object's name. However, facilitation in object recognition occurred with prior viewing of a different picture with the same name. This facilitation was not as great as that found for previously viewed pictures. C. Warren and Morton took these results as evidence for priming at the level of feature detectors, which resulted in more rapid acquisition of primed features. New instances of previously viewed objects share some features with the old picture; acquisition of these fea- 
tures is therefore facilitated. All of the features of previously viewed pictures are primed, and so greater facilitation occurs for these pictures. The authors thus argued that priming can affect the acquisition of visual information from pictures. However, as they themselves indicated, this conclusion is only one of several possible interpretations of the results.

\section{Paradigm for Studying \\ Information-Acquisition Effects}

In our experiments we investigated the effects of priming on acquisition of visual information from pictures. In our experimental paradigm, one can interrupt perceptual processing of primed and unprimed pictures at various points by varying target exposure duration and masking the targets. Probability of correct identification or recognition memory performance can then be used as a measure of how much information was acquired from the target. Whereas Ratcliff and McKoon (1978) and others varied priming at retrieval time but not encoding time, we did the opposite: We varied priming at encoding time but not retrieval time. Performance differences between primed and unprimed stimuli of equal duration must therefore reflect encoding differences.

\section{Performance Curves to Chart the Course of Information Acquisition}

In our first three experiments, we used a paradigm in which primed and unprimed pictures were presented for varying durations and followed by a mask. In Experiment 1, immediate target identification performance was measured for pictures of simple objects. In Experiment 2, pictures of objects were presented in the study phase of a recognition experiment; a yes/no recognition test followed, in which distractors had the same names as the targets. Experiment 3 was similar to Experiment 2, except that targets were complex, naturalistic scenes rather than pictures of objects. In all three experiments, primes were the category names of the targets. For instance, in Experiments 1 and 2, if the target was a dog, the appropriate prime was the word dog. In Experiment 3, if the target was a graveyard scene, the prime was the word graveyard. Thus in all three experiments, primed were category names but were still at the same hierarchical level as the targets.

From our paradigm we obtained performances for primed and unprimed stimuli as functions of exposure duration. We refer to these functions as performance curves. We assumed that performance is monotonically related to information acquired from the targets; given this assumption, performance curves reflect the temporal course of information acquisition from the target pictures. Critical for our subsequent reasoning is that if this monotonicity assumption is true, then equal values of acquired information imply equal values of memory performance, and vice versa.

\section{Two Models of How Priming Affects Information Acquisition}

Comparison of performance curves for primed and unprimed conditions allows us to test two classes of models of how primes exert their effect. According to the first model, having a prime is equivalent to having a brief glimpse of the target itself. According to the second model, having a prime increases the rate of extracting information from the target. We refer to these models as additive and multiplicative models.

\section{Additive Models}

According to an additive model, provision of a prime allows the same kind of processing that would otherwise be undertaken during some period, $k \mathrm{~ms}$, of a target picture's physical presence. Accordingly, information that is acquired in $d$ ms from a primed picture is acquired in $d+k \mathrm{~ms}$ from an unprimed picture. The additive equation relating acquired information from primed and unprimed pictures is therefore

$$
I_{P}(d)=I_{N}(d+k),
$$

where $I_{P}$ and $I_{N}$ represent information acquired from primed and unprimed pictures after exposure duration $x$, and $k$ is the equivalent exposure duration contributed by the prime. Given the monotonicity assumption that we just described, this equation for acquired information implies a corresponding equation for performance,

$$
P_{p}(d)=P_{N}(d+k)
$$

where $P_{P}(X)$ and $P_{N}(X)$ denote primed and unprimed performance after exposure duration $x$. Equation $1 \mathrm{~b}$ is graphically presented in Figure 1, top panel; the crucial prediction is that primed and unprimed performance curves are separated by a constant horizontal difference of $k \mathrm{~ms}(k=100 \mathrm{~ms}$ in this example). We characterize such curves as horizontally parallel.

Morton $(1964,1969)$ proposed such a model to explain context effects on word recognition; Seymour (1973) applied the model to explain picture recognition. According to the model, lexical memory consists of a set of activation-summing units called logogens. Both prime- and target-based information contribute to activation of logogens. When a logogen's activation level rises above some threshold level, that logogen "fires" and identification occurs. Rather than affecting the rate at which information is acquired from the physical stimulus, the prime is seen as contributing to activation. For brief exposures, the amount of activation from the target stimulus is assumed to increase monotonically with target exposure duration. Thus to reach any accuracy level, a shorter exposure is required for primed than for unprimed stimuli because some activation has already been provided by the prime. Because the activation provided by the prime is not qualitatively different from the activation provided by the target, presentation of the prime is equivalent to a brief exposure of the target, and the model thus falls into the class of additive models.

\section{Multiplicative Models}

According to a multiplicative account, provision of a prime increases the rate at which target information is acquired. If 

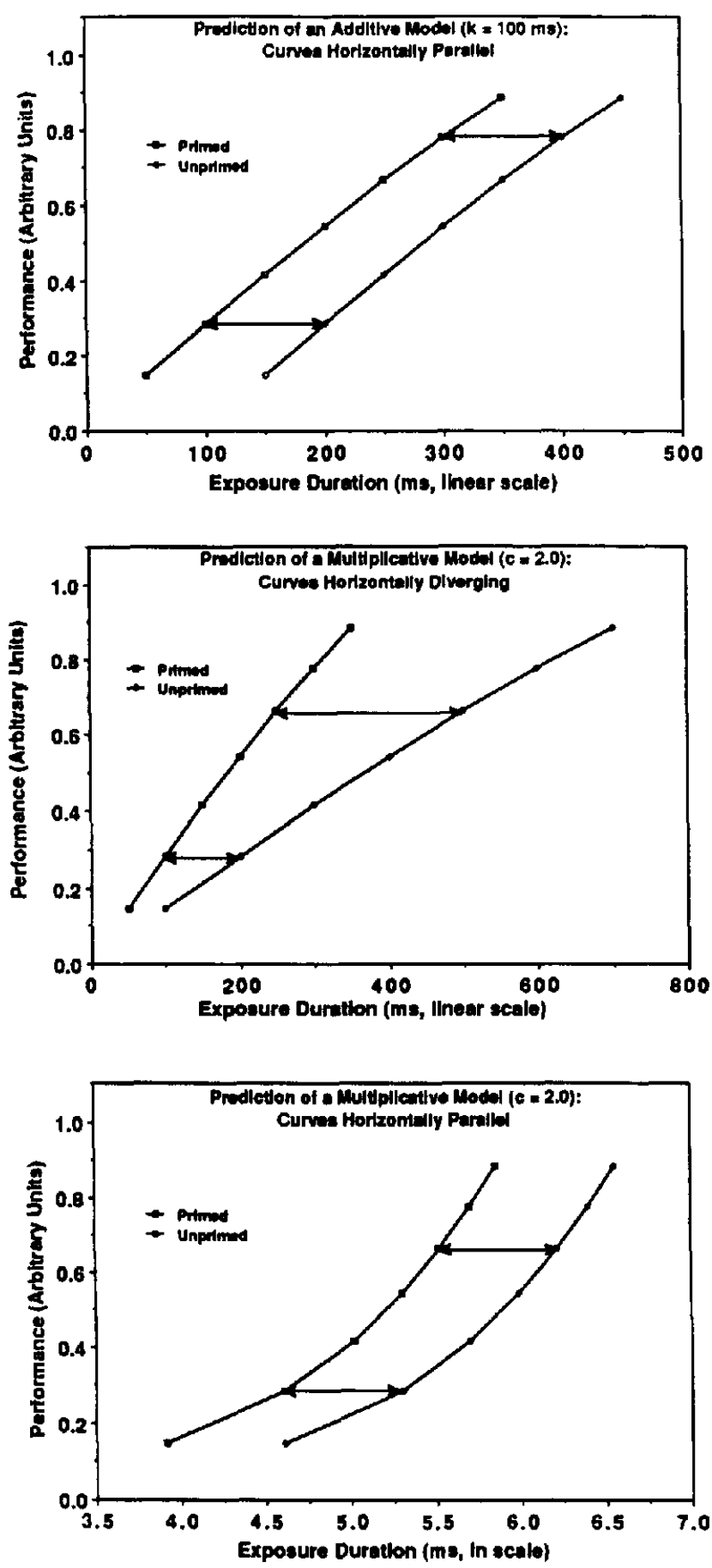

Figure I. Predictions of an additive model (top panel) and a multiplicative model (middle and bottom panels).

the rate increases by a factor of $c$, information that is acquired in $d \mathrm{~ms}$ from a primed picture is acquired in $c d \mathrm{~ms}$ from an unprimed picture. The same logic that went into the derivation of Equation $1 \mathrm{~b}$ implies that the multiplicative equation relating primed and unprimed performance is

$$
P_{P}(d)=P_{N}(c d)
$$

Equation 2 is graphically presented in Figure 1, middle panel; primed and unprimed curves diverge horizontally. This divergence is quite specific: The duration needed to reach any given performance level is greater by a constant factor $(c)$ for unprimed than for primed curves ( $c=2.0$ in this example).

One can conveniently represent (and test) the prediction of a multiplicative model by plotting performance as a function of $\log$ rather than linear duration. Because equal ratios in linear coordinates correspond to equal distances in log coordinates, the prediction of the multiplicative model is that the primed and unprimed performance curves are horizontally parallel when plotted as a function of log exposure duration. The equation relating the two performance curves becomes

$$
P_{P}[\ln (d)]=P_{N}[\ln (d)+\ln (c)] \text {. }
$$

This equation is graphically presented in Figure 1, bottom panel; in the middle panel, data are replotted as functions of $\log$, duration. The crucial prediction is that primed and unprimed curves are horizontally parallel, separated by a constant horizontal difference of $\ln (c)$.

\section{On the Generality of Additive and Multiplicative Predictions}

In summary, additive models of priming effects on picture encoding predict that primed and unprimed performance curves will be horizontally parallel on a linear exposure duration axis, whereas multiplicative models predict that these curves will diverge on a linear-duration axis and will be horizontally parallel on a log-duration axis.

It is important to note that these predictions are invariant over all monotonic transformations of the dependent variable (Loftus, 1978). This is because the predictions involve horizontal comparisons of performance curves-that is, comparisons of primed and unprimed durations that produce equal performance. Any set of points that are equal in one scale must also be equal in a monotonically transformed scale. This means that any conclusions issuing from our intended performance-curve comparisons (e.g., that curves are horizontally parallel on a linear- or a log-duration axis) will apply to any theoretical construct assumed to be montonically related to performance (e.g., amount of information acquired from the target).

The advantage of this theoretical machinery is that we do not need to make any strong assumptions concerning the scaling of the performance axis (e.g., that performance is exponentially related to acquired information). We do not even need to make assumptions about the specific nature of visual information. We need assume only that performance is monotonically related to the amount of information acquired from the target. The additive and multiplicative predictions that we have described enable us to avoid committing ourselves to very strong assumptions, and at the same time they allow us to draw strong conclusions about the nature of priming effects. Furthermore, if we find that primes exert, say, a multiplicative effect on encoding, this allows us to rule out an entire class of (additive) models rather than simply a single (strong) model. 
An alternative approach for distinguishing between additive and multiplicative models involves using response time as the dependent measure. It is then possible to generate speedaccuracy trade-off curves for the various experimental conditions (e.g., Dosher, 1976, 1984). In this paradigm, subjects are asked to make a response by some deadline after stimulus onset; response probability as a function of deadline time constitutes a speed-accuracy trade-off curve. Specific functions (typically exponentials) are fit to these curves, and the nature of underlying processes are inferred from the best fitting values of the function parameters. A speed-accuracy trade-off curve is conceptually similar to the performance curves that we use in our research; both represent performance as a function of available processing time.

Investigators using the speed-accuracy trade-off paradigm typically allow three free parameters in their theoretical speed-accuracy trade-off functions: a start parameter (i.e., the duration at which performance rises above chance), a function rate, and a function asymptote. A focal variable can then be examined in terms of which of these parameters the variable affects. There are close correspondences between such functions and our theoretical performance functions: A finding that the focal variable affects only rate corresponds to our finding of a multiplicative effect, and a finding that the focal variable affects only start duration corresponds to our finding of an additive effect. However, our equivalence analyses do not require the strong assumption of an underlying exponential function.

\section{Experiments}

We conducted four experiments. The first purpose of the experiments was to demonstrate priming effects on early visual information acquisition. The second purpose was to begin investigating the nature of such priming effects.

\section{Experiment 1}

In Experiment 1 we examined whether primes affect performance in an object-identification task. Primed and unprimed pictures of target objects were presented for durations ranging from 30 to $90 \mathrm{~ms}$. Kroll and Potter (1977) showed that pictures of objects are effectively primed by their names; accordingly, we used object names as primes. An object's name is of course a category name. Therefore, in this experiment (as in those that follow), primes were category names but were still at the same hierarchical level as the targets. A target object was preceded by a row of $X s$ (no-prime, or NP, condition), by the target's name (appropriate-prime, or AP, condition), or by the name of some semantically unrelated object (inappropriate-prime, or IP, condition). The subjects' task was to write down the name of the target object immediately after seeing it.

The test between the additive and multiplicative accounts of encoding effects involves examining the relation between the AP and the NP conditions; thus these conditions were of primary interest. The IP condition was included to prevent subjects from always naming the prime and to provide a measure of the guessing rate.

\section{Method}

Subjects. One hundred forty-four University of Washington undergraduates participated for class credit. They were tested in twentyfour groups of 5-8 subjects each.

Stimuli. Seventy-two color slides of common objects on grey backgrounds served as target stimuli. They were chosen in such a way that as many semantic categories as possible were represented in order to minimize intertarget priming effects. For each of the 72 objects, a priming slide containing the name of that object was prepared. An additional priming slide contained a row of four $X \mathbf{s}$. A noise mask consisted of a blank slide with a jumble of straight and curved black lines. The mask was presented at a luminance such that when a target object and a mask were presented simultaneously, the target could not be seen.

Apparatus. All slides were shown through Kodak random-access projectors. Subjects sat in a dimly lit room, and the stimuli were presented on a white wall. Target objects subtended a visual angle that ranged from $7.5^{\circ}$ to $11^{\circ}$ both vertically and horizontally, depending on the observer's position. The primes subtended a visual angle of $2^{\circ}-3^{\circ}$ vertically and $5^{\circ}-15^{\circ}$ horizontally, depending on the observer's position and the number of letters in the prime. The mask subtended a visual angle of $10^{\circ}-15^{\circ}$ vertically and $15^{\circ}-22^{\circ}$ horizontally. Exposure durations were controlled by Gerbrands tachistoscopic shutters that had rise and fall times of approximately $1 \mathrm{~ms}$. All display equipment was enclosed in a soundproof box and was under control of an Apple II computer system described by Loftus, Gillispie, Tigre, and Nelson (1984).

Design and procedure. The four possible exposure durations of the target objects were $30,50,70$, and $90 \mathrm{~ms}$. The three priming conditions were factorially combined with exposure duration. This design resulted in a total of 12 conditions. Twenty-four groups of subjects were tested. Each group had 36 trials, and so only half of the target objects were presented in a single experimental session. In 12 of the 24 groups, subjects saw only targets that were in odd-numbered slots in the slide tray, and subjects in the other 12 groups saw only targets that were in even-numbered slots. Each group saw three targets in each of the 12 conditions. With this restriction, conditions were presented randomly over the 36 trials.

The sequence of events for each trial was as follows. First a 500ms tone signaled the start of a trial. A prime was then presented for $2 s$ and followed by a target presented for one of the four exposure durations and then by the mask. The mask remained on for $10 \mathrm{~s}$, providing the subjects with enough light to write down their responses. The next trial began at the offset of the mask. Subjects were informed that word primes had a 50:50 chance of naming the upcoming target object.

Each object picture served once in each of the 12 experimental conditions over the 12 groups for which it appeared as a target.

\section{Results and Discussion}

Target identification probabilities for all 12 conditions are presented in Table 1, columns 2, 4, and 5 (labeled "Uncorrected AP Condition," "IP Condition," and "NP Condition"). The standard error of all means is 0.028 . The subjects could, if they wished, guess the identification of an appropriateprimed target simply by naming the prime. We term this strategy a guessing strategy and determined the probability of using it by calculating the probabilities of incorrectly naming the prime in the unrelated-prime condition for the four exposure durations (see Table 1, column 6). 
Table 1

Identification Probabilities: Experiment $]$

\begin{tabular}{|c|c|c|c|c|c|}
\hline $\begin{array}{c}\begin{array}{c}\text { Exposure } \\
\text { duration } \\
\text { (ms) }\end{array} \\
\end{array}$ & $\begin{array}{l}\text { Uncor- } \\
\text { rected } \\
\text { AP } \\
\text { condition }\end{array}$ & $\begin{array}{c}\text { Corrected } \\
\text { AP } \\
\text { condition } \\
\end{array}$ & $\begin{array}{c}\text { IP } \\
\text { condition }\end{array}$ & $\begin{array}{c}\mathrm{NP} \\
\text { condition }\end{array}$ & $\begin{array}{c}\text { Guessing } \\
\text { rate }\end{array}$ \\
\hline 30 & .457 & .407 & .076 & .114 & .084 \\
\hline 50 & .799 & .783 & .436 & .474 & .073 \\
\hline 70 & .923 & .919 & .676 & .727 & .055 \\
\hline 90 & .924 & .922 & .779 & .802 & .025 \\
\hline
\end{tabular}

Note. $\mathrm{AP}=$ appropriate prime; $\mathrm{IP}=$ inappropriate prime; $\mathrm{NP}=$ no prime. The probabilities for the 12 conditions are given in columns 3, 4, and 5. Estimated guessing probabilities are for the AP condition. Data points in columns 2,4 , and 5 are based on 432 observations. Standard error is 0.028 .

An analysis of variance (ANOVA) was run on the 12 conditions. Because there were unequal numbers of subjects in the various groups, we used groups as the unit of analysis to preserve an unbiased counterbalancing scheme. The main effects of priming condition, $F(2,46)=89.47$, and exposure duration, $F(3,69)=210.80$, were significant. Although NP performance was slightly better than IP performance, the difference between the two was not statistically significant, $t(23)=0.80$. We tentatively concluded that this finding indicates no cost of an unrelated prime; this conclusion was confirmed in Experiments 2-4.

It is quite evident that an appropriate prime substantially increased performance: For AP performance versus NP performance, $t(23)=5.35$, and for AP performance versus IP performance, $t(23)=6.15$.

We next corrected the AP identification probabilities for guessing, using the equation

$$
p^{\prime}=(p-g) /(1-g)
$$

where $p^{\prime}$ is the corrected probability, $p$ is the original probability, and $g$ is the guessing rate for that exposure duration. These corrected AP probabilities are shown in Figure 2 (top panels) along with the mean of the IP and NP probabilities. To test additive and multiplicative models, we plotted the

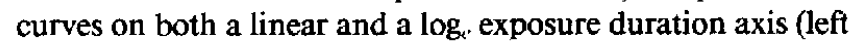
and right top panels). For ease of discourse in this and subsequent experiments, we refer to the AP performance curve as the primed curve and to the mean of the IP and NP curves as the unprimed curve.

The superiority of the primed curve to the unprimed performance curve indicates that primes affect picture encoding. Can this effect be described by either an additive or a multiplicative model? As described earlier, the crucial tests involve determining whether there exists a constant horizontal distance between the AP and NP performance curves when these were plotted on a linear or log exposure duration axis. To assess such relations, we shifted the primed curve horizontally in relation to the unprimed curve in such a way as to provide the best monotonic function relating all eight (primed and unprimed) points to exposure duration (see bottom panels of Figure 2). In the left panel, this shift is by the best linear duration $(k=37 \mathrm{~ms})$, and in the right panel, the shift is by the best $\log$ duration $(0.45 \log$ units, which corresponds to $c=e^{0.45}=1.57$ ).
It is evident that a log shift allows a perfect monotonic relation, whereas there is no linear shift that produces a monotonic relation between performance and exposure duration. Although we do not provide formal statistical tests of these relations, any vertical rearranging of the additive-shift data points to bring them into a monotonic configuration would require moves of many standard deviations. This constitutes informal statistical evidence against the additive model's fit. Accordingly, we tentatively conclude that a multiplicative model best describes the priming effect and thereby suggest that priming an object with its category name speeds up information acquisition.

\section{Experiment 2}

The results of Experiment 1 indicate that primes speed the encoding process. This conclusion depends, however, on the assumption of equal guessing rates in the IP and AP conditions.

There is an alternative explanation: The IP guessing rates underestimate the AP guessing rates. The reason is that any visual information acquired about the target in the IP conditions is likely to disconfirm the hypothesis that the prime names the target, whereas information acquired about AP targets is likely to be confirming evidence. The comparison between performance curves depends, of course, on accurately estimating performance. In Experiment 2, therefore, we replicated the results of Experiment 1, using a paradigm that eliminated the possibility of differential response bias between conditions. In Experiment 2, as in Experiment 1, primed and unprimed targets were presented, randomly intermingled, during the study phase. Memory for the pictures was then measured in a later yes-no recognition test. This procedure rules out a response-bias explanation for the results of Experiment 1 because all stimuli-both primed and unprimedwere treated identically during the test phase.

We had a second goal in Experiment 2, which was to determine whether priming effects could be found on specifically visual information acquisition rather than on memory for names of identified target objects. Accordingly, subjects saw six objects belonging to each of twelve object categories during study; for instance, six fish were presented, six cars were presented, and so on, in a random order. Targets were preceded by their category name on AP trials, by the name of one of the other categories on IP trials, and by a string of five $X$ s on NP trials. During the test, targets were randomly mixed with distractors consisting of six additional exemplars of each category. Thus if primes facilitate recognition performance, they must do so by affecting the amount of visual information that is acquired from the targets.

\section{Method}

Subjects. Three hundred eighty-four University of Washington undergraduates participated for class credit. They were run in seventytwo groups of 4-5 subjects each.

Stimuli. One hundred forty-four color slides of common objects on gray backgrounds served as target stimuli. The objects represented 12 instances of each of 12 categories: shoe, glove, dog, phone, clock, 

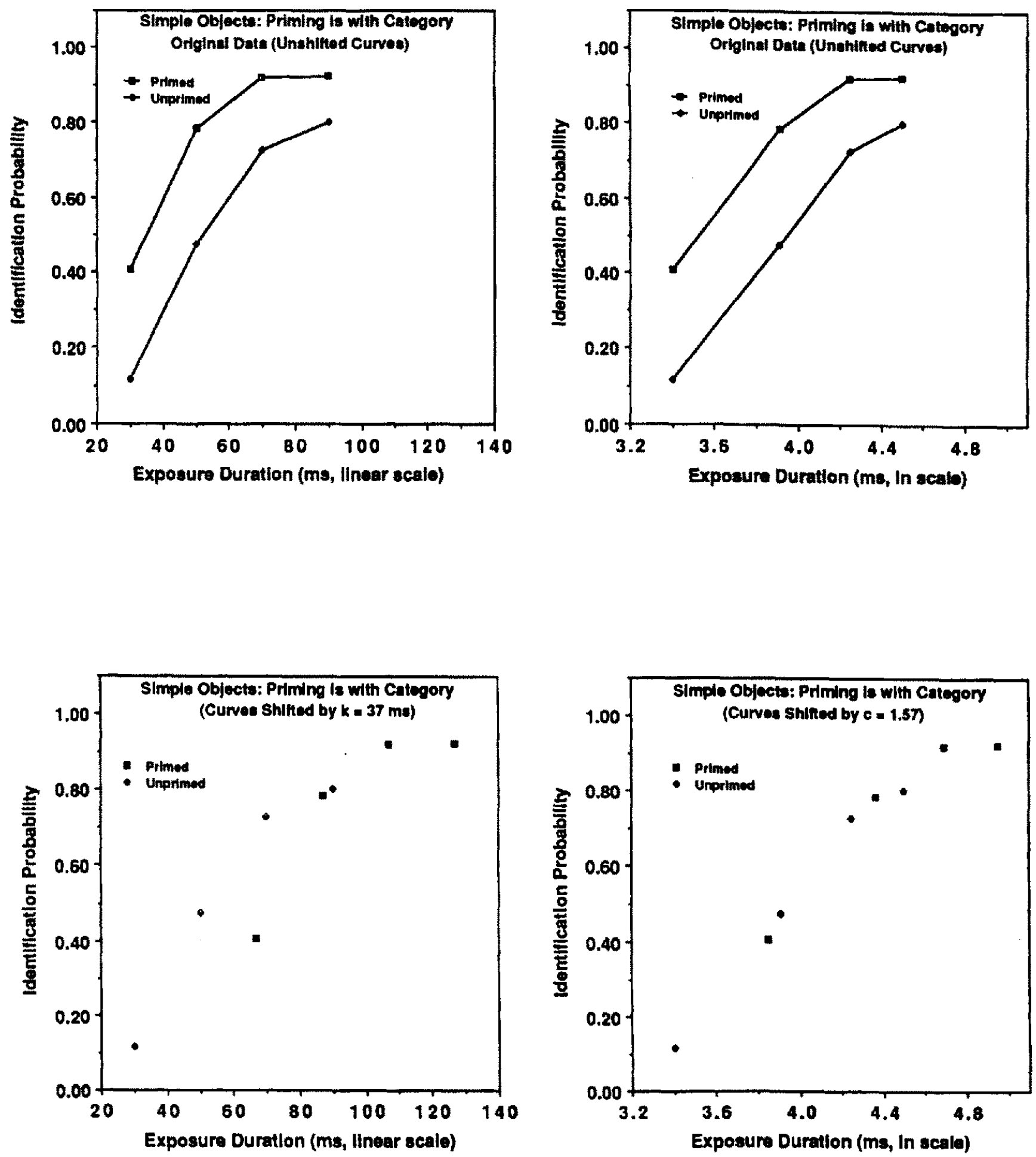

Figure 2. Experiment 1 performance curves: Object identification probability as a function of exposure duration for primed and unprimed objects. (Top panels show original data, and bottom panels show primed curve shifted to the right so as to provide appropriate overlap with the unprimed curve. Exposure duration is on a linear scale in the left panels and on a $\log _{e}$ scale in the right panels.)

lamp, fish, chair, key, cup, ball, and car. The slides were placed in two stimulus trays. Each stimulus tray contained 72 slides and included 6 instances of each category.

Thirteen priming slides were prepared: one of each category name, in capital letters, plus one consisting of a row of five $X \mathrm{~s}$. The mask was the same one used in Experiment 1. The mask was presented at a luminance such that when a target object and mask were presented simultaneously, the target could not be seen.

Apparatus. Subjects sat in a dimly lit room, and the stimuli were presented on a white screen. Target objects subtended a visual angle 
that ranged from $2^{\circ}$ to $23^{\circ}$ horizontally and from $2^{\circ}$ to $16^{\circ}$ vertically, depending on the particular object and the observer's position. Primes subtended a visual angle that ranged from $2.5^{\circ}$ to $6^{\circ}$ horizontally and from $0.7^{\circ}$ to $1.2^{\circ}$ vertically. Kodak standard projectors were used to present the target, mask, and fixation point slides. Priming slides were presented with a Kodak random-access projector. All display equipment was enclosed in a soundproof box. All display and response apparatus was controlled by an XT-compatible computer system described by Stoddard and Loftus (1988).

Design and procedure. An experimental session entailed a study phase and a test phase for each of the two stimulus trays in succession.

A study phase consisted of 36 trials. Each trial fell into one of eighteen experimental conditions defined by six exposure durations combined with three priming conditions. Stimulus duration was 40 , $80,120,160,200$, or $240 \mathrm{~ms}$. The three priming conditions were as follows: a target was preceded by its category name (AP condition), by the name of some unrelated object (IP condition), or by a row of $X$ s (NP condition). Each of the conditions occurred twice over the 36 trials. Three objects from each category appeared, one in each of the priming conditions and in three of the six exposure durations.

The sequence of events for each trial was as follows. A fixation point and tone were presented for $500 \mathrm{~ms}$, signaling the start of each trial. A prime was presented for $250 \mathrm{~ms}$ and was followed first by a 400-ms blank (dark) delay, then by a target presented for one of the six exposure durations, and then by the mask. The stimulus onset asynchrony (SOA) between trials was $4 \mathrm{~s}$. Subjects were told that there was a $50: 50$ chance that a word prime would correctly name the upcoming target.

An old/new recognition task immediately followed the 36 study trials. Of the 72 pictures in each tray, 36 were targets and the other 36 were distractors. The ordering of target/distractor was random. Subjects indicated whether each object was old or new by pressing the appropriate button on a response box. The next picture appeared after all subjects in the group had responded. After the 72 trials, the entire procedure was repeated for the second slide tray. Over all 72 groups, every picture appeared twice in each of the study conditions and appeared equally often as a target and a distractor.

\section{Results and Discussion}

Because all conditions were randomly intermingled during the study phase, there was only a single false-alarm probability, which was 0.291 . Hit probabilities for all 18 conditions are provided in Table 2 . The standard error of all means is 0.013 . The effects of exposure duration, $F(5,1615)=250.68$, and priming condition, $F(2,646)=25.14$, were both significant.' The AP performances differed significantly from both the NP and the IP performances, $t \mathrm{~s}(323)=38.92$ and 32.40 . Performances in the IP and NP conditions were almost iden-

Table 2

Hit Probabilities: Experiment 2

\begin{tabular}{cccc}
\hline $\begin{array}{c}\text { Exposure } \\
\text { duration (ms) }\end{array}$ & $\begin{array}{c}\text { AP } \\
\text { condition }\end{array}$ & $\begin{array}{c}\text { IP } \\
\text { condition }\end{array}$ & $\begin{array}{c}\text { NP } \\
\text { condition }\end{array}$ \\
\hline 40 & .447 & .409 & .412 \\
80 & .678 & .619 & .605 \\
120 & .691 & .669 & .668 \\
160 & .736 & .672 & .682 \\
200 & .750 & .715 & .701 \\
240 & .781 & .730 & .744 \\
\hline
\end{tabular}

Note. $\mathrm{AP}=$ appropriate prime; $\mathrm{IP}=$ inappropriate prime; $\mathrm{NP}=$ no prime. Data points are based on 1,944 observations. Standard error is 0.013 . tical; for the main effect of prime, $t(323)<1$, and for the Duration $\times$ Prime interaction, $F(5,1615)<1$. This confirms the Experiment 1 finding of no cost for the IP conditions. For the remaining analyses, the data from these two conditions were pooled to produce a single unprimed performance curve that we compared with the AP, or primed, performance curve.

The significant priming effect indicates that primes affect recognition performance. Given the design of Experiment 2, we can tentatively conclude from this result that preceding a picture of an object by its category name affects early visual processing and, in particular, acquisition of information from the picture that is primarily visual. We now turn to the issue of whether this effect can be described by a multiplicative or an additive model. Recall that in Experiment 1, a multiplicative model best described the priming effect.

In Figure 3, which is organized in the same way as Figure 2 , primed and unprimed performance curves are plotted as functions of exposure duration on linear and $\log$ scales (top panels). In the bottom panels, the primed curve has been shifted right in relation to the unprimed curve so as to provide the best monotonic function relating all 12 points to exposure duration. In the left panel, this shift is by the best linear duration ( $k=55 \mathrm{~ms}$ ), and in the right panel, the shift is by the best $\log$ duration $\left(0.40 \log _{c}\right.$ units, which corresponds to $c=e^{0.40}=1.49$ ). Shifting by a constant factor provided a somewhat better fit; as in Experiment 1, a log shift produced a perfect monotonic relation between performance and exposure duration, whereas there was no linear shift that produced perfect monotonicity. This finding supports the proposition that priming an object with its category name speeds up information acquisition. However, we argue in the General Discussion that the process is somewhat more complicated.

\section{Experiment 3}

At a general level, Experiments 1 and 2 have demonstrated that primes affect information acquisition from photographs of objects. The purpose of Experiment 3 was to extend this finding to another class of pictorial stimuli: naturalistic scenes. The Experiment 2 paradigm was used; however, stimuli were scenes rather than objects, and primes were the names of scene categories (e.g., supermarket) rather than object categories (in other words, primes named the gists of the scenes).

Priming effects for scene recognition would be interesting for a number of reasons. From an ecological perspective, much of vision involves scene perception. Moreover, scenes are usually primed in some way. People rarely find themselves confronted with completely unexpected scenes; rather, they generally have prior knowledge about the scenes that they are going to encounter. Mountain climbers expect to see mountain vistas, baseball fans expect to see baseball diamonds, and so on. From a theoretical perspective, gist is important for

\footnotetext{
${ }^{1}$ Subjects, not groups, were used as the analysis unit. There were 72 groups, which represented two 36-group replications of the experimental design. There were 5 subjects in each of the first-replication groups, and 4 subjects in each of the second-replication groups. Accordingly, we could perform the analysis on individual subjects without biasing the counterbalancing scheme.
} 

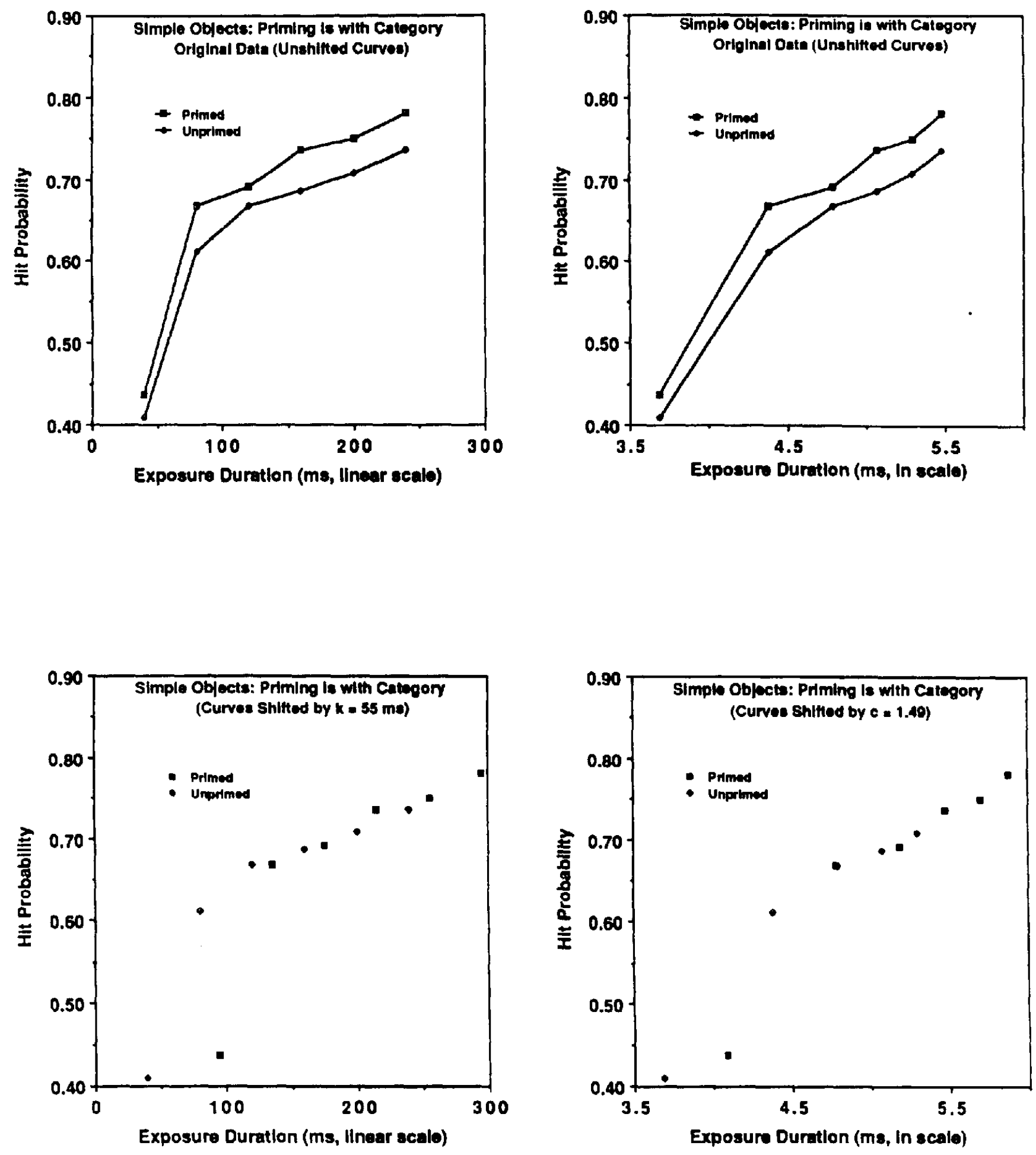

Figure 3. Experiment 2 performance curves: Hit probability as a function of exposure duration for primed and unprimed objects. (Top panels show original data, and bottom panels show primed curve shifted to the right so as to provide appropriate overlap with the unprimed curve. Exposure duration is on a linear scale in the left panels and on a $\log _{e}$ scale in the right panels.)

picture perception. When scenes are jumbled so that they have no inherent gist, memory for the scene is poor and search time for objects contained in the scene are long (Biederman et al., 1973). When subjects are presented a naturalistic picture, without prior knowledge of its content, the first eye fixation on the scene is designed to extract the picture's gist, whereas subsequent fixations are designed to encode specific details (e.g., Loftus et al., 1983). 


\section{Method}

Subjects. Two hundred ten University of Washington undergraduates participated for class credit. They were run in thirty groups of 5-8 subjects each.

Stimuli. One hundred twenty color slides of naturalistic scenes served as target stimuli. The scenes represented 12 instances of each of 10 categories: supermarket, playground, alley, mountains, classroom, library, bathroom, houses, graveyard, and parking lot. Categories were chosen to be as different from one another as possible to avoid intercategory priming. The slides were placed in two stimulus trays. Each stimulus tray contained 60 slides and included 6 instances of each category.

Eleven priming slides were prepared: one of each category name, in capital letters, plus one consisting of a row of five $X \mathrm{~s}$. The noise mask was the same one used in Experiments 1 and 2. The mask was presented at a luminance such that when a target scene and mask were presented simultaneously, the target could not be seen.

Apparatus. Stimuli were presented with the same apparatus as in Experiment 1. Subjects sat in a dimly lit room, and the stimuli were presented on a white wall. Target scenes subtended a visual angle that ranged from $15^{\circ}$ to $22^{\circ}$ horizontally and from $10^{\circ}$ to $15^{\circ}$ vertically, depending on the observer's position. Primes subtended a visual angle that ranged from $4^{\circ}$ to $15^{\circ}$ horizontally and from $2^{\circ}$ to $3^{\circ}$ vertically, depending on the prime's length and the observer's position. Kodak standard projectors were used to present the target, mask, and fixation point slides. Priming slides were presented with a Kodak randomaccess projector.

Design and procedure. The design and procedure were very similar to those of Experiment 2. An experimental session entailed a study phase and a test phase for each of the two stimulus trays in succession.

A study phase consisted of 30 trials. Each trial fell into one of fifteen experimental conditions defined by five exposure durations combined with three priming conditions. Stimulus duration was 50 , $70,90,110$, or $130 \mathrm{~ms}$. The three priming conditions were as follows: A target was preceded by its category name (AP condition), by some incorrect category name (IP condition), or by a row of $X \mathbf{s}$ (NP condition). Each of the conditions occurred twice over the 30 trials. Three objects from each category appeared, one in each of the priming conditions and in three of the five exposure durations.

The sequence of events for each trial was as follows. A fixation point and tone were presented for $500 \mathrm{~ms}$, signaling the start of each trial. A prime was presented for $250 \mathrm{~ms}$ and was followed first by a 400-ms blank (dark) delay, then by a target presented for one of the six exposure durations, and then by the mask. The SOA between trials was $4 \mathrm{~s}$. Subjects were told that there was a 50:50 chance that a word prime would correctly name the upcoming scene.

An old/new recognition task immediately followed the 30 study trials. Of the 60 pictures in each tray, 30 were targets and the other 30 were distractors. The ordering of target/distractor was random. Subjects indicated whether each scene was old or new by pressing the appropriate button on a response box. The next picture appeared after all subjects in the group responded. After the 60 trials the entire procedure was repeated for the second slide tray. Over all 30 groups, every picture appeared once in each of the study conditions and appeared equally often as a target and a distractor.

\section{Results and Discussion}

The false-alarm probability was 0.323 . Hit probabilities for all 15 conditions are provided in Table 3 . The standard error of all means is 0.017 . The effects of exposure duration, $F(4$, $836)=58.37$, and priming condition, $F(2,418)=5.50$, were
Table 3

Hit Probabilities: Experiment 3

\begin{tabular}{cccc}
\hline $\begin{array}{c}\text { Exposure } \\
\text { duration (ms) }\end{array}$ & $\begin{array}{c}\text { AP } \\
\text { condition }\end{array}$ & $\begin{array}{c}\text { IP } \\
\text { condition }\end{array}$ & $\begin{array}{c}\text { NP } \\
\text { condition }\end{array}$ \\
\hline 50 & .541 & .497 & .481 \\
70 & .602 & .546 & .563 \\
90 & .641 & .633 & .611 \\
110 & .695 & .652 & .652 \\
130 & .723 & .726 & .688 \\
\hline
\end{tabular}

Note. $\mathrm{AP}=$ appropriate prime; IP = inappropriate prime; $\mathrm{NP}=$ no prime. Data points are based on 1,260 observations. Standard error is 0.017 .

both significant. Performance in the AP condition differed significantly from those in both the NP and IP conditions, $t(209)=2.80$ and 2.00. Performances in the IP and NP conditions were almost identical; for the main effect, $t(209)<$ 1 , and for the interaction, $F(2,418)<1$. As in Experiment 2, the data from these two conditions were pooled for the remaining analyses to produce a single unprimed performance curve that we compared with the AP, or primed, performance curve.

Using the same logic as in Experiments 1 and 2, we can conclude that preceding a naturalistic scene by its gist affects acquisition of information from the picture that is primarily visual. Can this effect be described by a multiplicative or an additive model?

In Figure 4, which is organized in the same way as Figures 2 and 3 , primed and unprimed performance curves are plotted as functions of exposure duration on linear and $\log _{c}$ scales. In the bottom panels, the primed curve has been shifted right in relation to the unprimed curve so as to provide the best monotonic function relating all 10 points to exposure duration. In the left panel, this shift is by the best linear duration ( $k=12 \mathrm{~ms}$ ), and in the right panel, the shift is by the best log duration $\left(0.157 \log _{e}\right.$ units, which corresponds to $c=e^{0.157}=$ 1.17). The two methods do not differ very much, although the fit appears somewhat smoother with the linear shift than with the log shift.

The absolute magnitudes associated with both shifts $(k=$ $12 \mathrm{~ms}$ and $c=1.17$ ) are quite small (no priming effect at all is associated with $k=0$ or $c=1.00$ ). The statistically significant priming effect indicates, however, that the difference, however viewed, is reliable.

\section{Experiment 4}

We have noted that the target-distractor configuration of Experiments 2 and 3 is such that verbally encoding an appropriate prime (e.g., encoding $d o g$ when a picture of a dog was upcoming) could not in and of itself improve subsequent memory. Accordingly, the most plausible and parsimonious explanation for the data of Experiments 1-3 is that provision of an appropriate prime affects subsequent memory performance through its effect on perceptual processing--that is, on acquisition of stimulus information.

Nonetheless, the data thus far do not allow us to unequivocally rule out the possibility that the prime affects some 

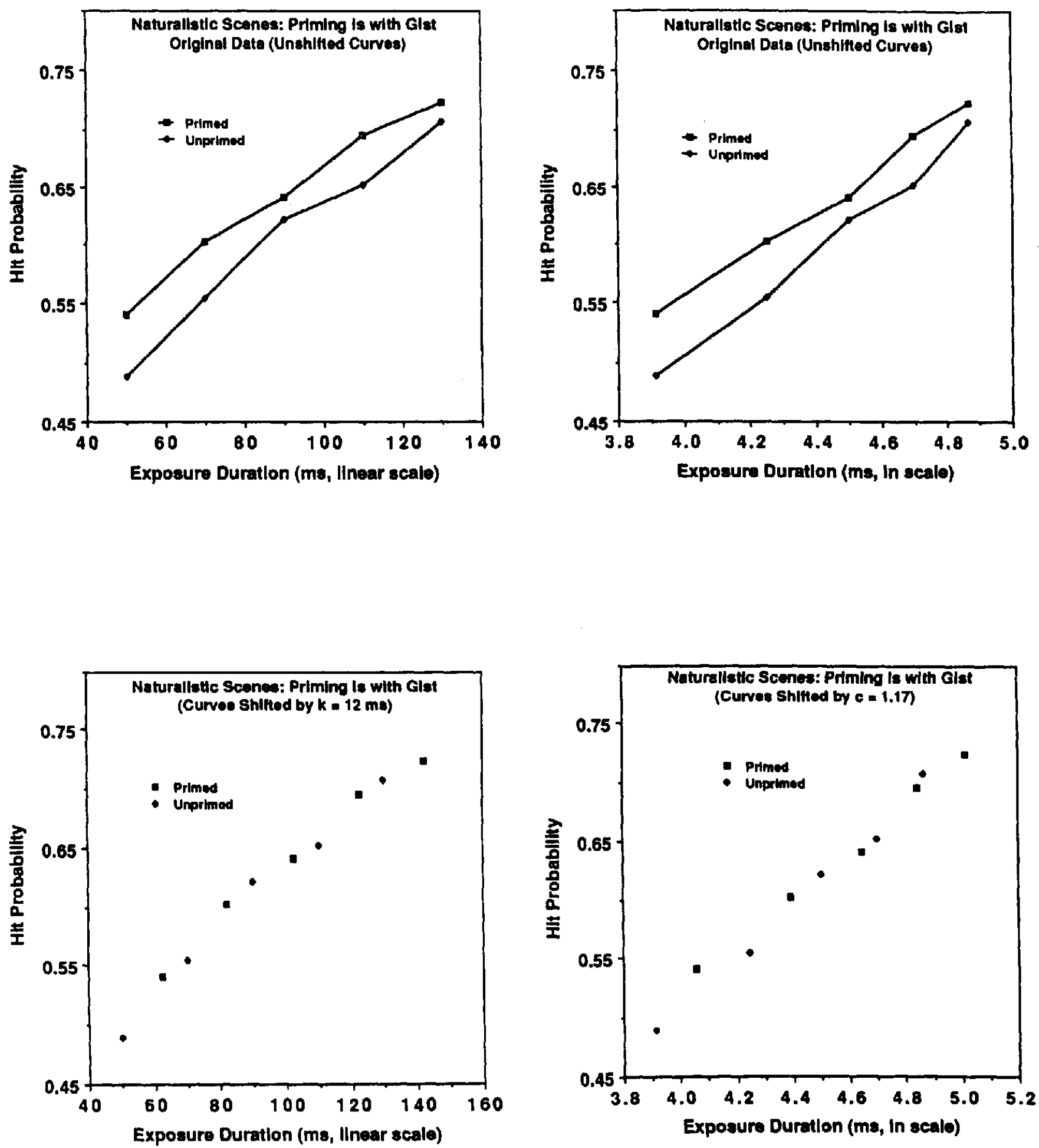

Figure 4. Experiment 3 performance curves: Hit probability as a function of exposure duration for primed and unprimed objects. (Top panels show original data, and bottom panels show primed curve shifted to the right so as to provide appropriate overlap with the unprimed curve.

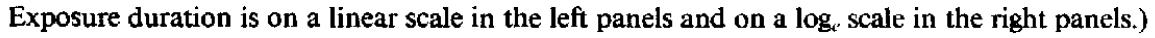

postperceptual rather than perceptual processing stage. Perhaps merely having an appropriate prime in close temporal contiguity with the target somehow improves memory for the target. Experiment 4 was designed to investigate this possibil- ity. Experiment 4 was similar to Experiment 2: Stimuli were simple objects, and the primes were objects' category names. In Experiment 4, however, primes were presented after, in addition to before, the target stimulus. If a prime affects a 
postperceptual stage, then an appropriate prime will improve memory performance in both prime-before and prime-after conditions.

Experiment 4 was a simple control experiment; unlike Experiments 1-3, it was not designed to investigate information acquisition. Accordingly, we used only a single exposure duration, $150 \mathrm{~ms}$. Given the results of Experiment 2 (see Table 2 and Figure 3), we expected that when the prime preceded the target, there would be a facilitatory effect of an appropriate prime, but no difference between an inappropriate and neutral prime. The principal question was whether this data pattern would also emerge when the prime followed the target.

\section{Method}

Subjects. Eighty-four University of Washington undergraduates participated for class credit. They were run in twelve groups of 7 subjects each.

Stimuli and apparatus. Stimuli and apparatus were those used in Experiment 2.

Design and procedure. As in Experiments 2 and 3, an experimental session entailed a study phase and a test phase on each of the two stimulus trays in succession.

A study phase consisted of 36 trials. Target duration on all trials was $150 \mathrm{~ms}$. Each trial fell into one of six experimental conditions defined by the three priming conditions (AP, IP, or NP) combined with prime preceding or following the stimulus. The three priming conditions occurred pseudorandomly across study trials within each study phase. However, whether prime occurred before or after was blocked by study phase: In one of the two study phases, the prime always preceded the target, whereas in the other study phase, the prime always followed the target. As in Experiment 2, three instances of each of 12 categories were shown during each study phase. One instance of each of the 12 categories fell into each priming condition within each study phase; hence within an experimental session, a single instance of each category was assigned to each of the six study conditions.

The sequence of events on each study trial was as follows. A fixation point and a tone were presented for $500 \mathrm{~ms}$ signaling the start of each trial. On a prime-before trial, this was followed by the prime for $250 \mathrm{~ms}$, a $400-\mathrm{ms}$ blank delay, the target for $150 \mathrm{~ms}$, and the 300 -ms mask. On a prime-after trial, the fixation point/tone was followed by the 150 -ms target, the 300 -ms mask, a 400 -ms blank delay, and a 250 -ms prime. There was always a 2,400-ms dark interstimulus interval between the end of the last trial event and the start of the beginning of the next trial (i.e., the trial-to-trial SOA was $4,000 \mathrm{~ms}$ ). Subjects were informed of the sequence of events, with emphasis on the prime-target temporal relation, before each study phase.

The test phase that followed each study phase was identical to that of Experiment 2. Over the twelve groups, each of the 144 total stimuli appeared once in each of the six study conditions and equally often as a target and distractor. Six groups participated in the prime-before study phase before the prime-after study phase; this order was reversed for the other six groups.

\section{Results and Discussion}

Because the prime's occurrence before or after was blocked by study phase, there was a false-alarm probability for each of the two before/after levels. The complete data of Experiment 4 are shown in Table 4 . The standard error around all
Table 4

False-Alarm and Hit Probabilities: Experiment 4

\begin{tabular}{lcccc}
\hline & & \multicolumn{3}{c}{ Hit probability } \\
\cline { 3 - 5 } $\begin{array}{c}\text { Prime } \\
\text { condition }\end{array}$ & $\begin{array}{c}\text { False-alarm } \\
\text { probability }\end{array}$ & $\begin{array}{c}\mathrm{AP} \\
\text { condition }\end{array}$ & $\begin{array}{c}\text { IP } \\
\text { condition }\end{array}$ & $\begin{array}{c}\text { NP } \\
\text { condition }\end{array}$ \\
\hline Prime before & .237 & .698 & .655 & .639 \\
Prime after & .280 & .614 & .630 & .615 \\
\hline
\end{tabular}

Note. AP = appropriate prime; IP = inappropriate prime; $\mathrm{NP}=$ no prime. False-alarm probabilities are based on 3,024 observations; hit probabilities are based on 1,008 observations. For the hit probabilities, standard error is 0.015 .

means is 0.015 . The false-alarm probability was greater and the hit probabilities were lower for the prime-after condition than for the prime-before condition, which indicates better performance for the prime-before condition. This main effect could have been due to any of a number of things, including the temporal position of the target within each study trial.

Of principal interest is the interaction between prime position and prime type. With respect to the sample means, it is evident that in the prime-before conditions, the pattern of results was as in Experiments 1-3: Performance was better in the AP condition than in the IP and NP conditions, whose performances in turn differed little from one another. In the prime-after conditions, however, there was very little difference among the three priming conditions.

A $3 \times 2$ (Prime Condition $\times$ Prime Before/After) ANOva on the hit rates supported these assertions. There was a significant interaction between prime position and prime type, $F(2,166)=3.22$. When the prime preceded the target, performance in the AP condition was statistically greater than the mean of the IP and NP performances, $t(83)=2.74$, whereas the IP and NP performances did not differ, $t(83)<$ I. This result, of course, is entirely consistent with the data of Experiments 1-3. In contrast, when the prime followed the target, there was no prime type effect, $F(2,166)<1$. The AP sample mean is actually a little less than either the IP or NP sample means. The statistical power is such that the probability is less than .05 that the AP condition population mean exceeds the mean of the IP and NP condition population means by more than 0.019 .

In short, if there were any facilitatory priming effect when the prime followed the target, it did not appear in our pattern of sample means, it was less than two percentage points, and it certainly could not account for the priming effects found in Experiments 1-3. We conclude that a prime does indeed have a perceptual effect: It improves acquisition of information from the target.

\section{General Discussion}

\section{Summary of Results}

Primes affected perceptual processing in all four experiments. However, the degree to which the priming effect could be unambiguously described by either an additive or a multiplicative model varied. We discuss these two assertions in turn. 


\section{Primes Affect Information Acquisition From Visual Stimuli}

Preceding a picture with its category name increased performance for both objects (Experiments 1, 2, and 4) and scenes (Experiment 3). In Experiment 1, immediate object identification was used as the response measure, a procedure that permits the possibility that the priming effect was mediated by response bias. In Experiments 2-4, however, delayed recognition was used as the response measure. Because stimuli in all three priming conditions were treated identically at test, performance differences can be attributed to the prime's effect on encoding processes. Moreover, in Experiments 2-4, distractors and targets came from the same semantic categories; thus the prime's effect can be further isolated to acquisition of visual, as opposed to semantic, information. Last, we demonstrated in Experiment 4 that the priming effects of Experiments 1-3 could not be ascribed to any sort of general memorial processes; they are specifically perceptual information-acquisition effects.

\section{Multiplicative Versus Additive Effects}

Can we be more specific in describing the prime's effect on information acquisition? Earlier, we described two general models that could potentially describe the effect of priming (or any other independent variable) on information acquisition. According to an additive model, providing a prime is equivalent to providing a $k$-ms glimpse of the target picture. According to a multiplicative model, providing a prime speeds up information acquisition by a factor of $c$. Experiments 1-3 differed in terms of how well the prime's effect could be described by these two models.

As indicated in Figure 2, the Experiment 1 data could be described essentially perfectly by a multiplicative model and only poorly by an additive model. As indicated in Figure 3, the Experiment 2 data could be described better by a multiplicative than by an additive model, although the additive fit was not particularly bad, particularly at long exposure durations. Last, as indicated in Figure 4, the Experiment 3 data could be described almost equally well by either model. In short, characterizing priming as simply an additive or a multiplicative effect would constitute an insufficient account of our data.

\section{Model of Picture Encoding}

We now describe a model that incorporates both multiplicative and additive effects and is intended to apply to both object and scene encoding.

The model's central idea is that regardless of whether the target is an object or a scene, the goals of visual processing are first to identify the target's category and then to distinguish the target from other instances of the same category. For example, when a picture of an apple is presented, the first goal is to identify it as an apple. The goal of subsequent processing is to provide information sufficient to distinguish this particular apple from other apples. Similarly, if a supermarket scene is presented, initial processing identifies it as a supermarket scene, whereas subsequent processing distinguishes it from other supermarket scenes. Put another way, the first goal of picture processing is to identify the type of the target. The subsequent goal is to provide representation of the target as a token (or specific exemplar). Appropriate primes identify the target type, regardless of whether the target is a scene or an object.

The model asserts that visual information is acquired more rapidly from pictures of a known category than from pictures of an unknown category. Primed targets constitute pictures of a known category because the category is provided by the prime. Information is therefore initially acquired more rapidly from primed than from unprimed pictures. However, this encoding advantage for primed pictures does not last forever. If an unprimed picture's duration is sufficiently long, its category will be identified as a normal result of visual processing. Once an unprimed picture's category has been identified, there is no longer any difference between primed and unprimed pictures. Both simply represent pictures of a known category.

As detailed in the next section, the model predicts multiplicative effects for short target durations and additive effects for long target durations. The hybrid nature of the model reflects (a) a two-stage model of visual processing (pre- and postcategory identification) and (b) our particular experimental conditions, which define the domain of the model. These include prime versus no prime, short versus long duration, and object identification (Experiment 1) versus forced-choice recognition (Experiments 2-4).

\section{Assumptions of the Model}

1. Category knowledge increases information-acquisition rate. Information is acquired more rapidly from pictures of a known category than from pictures of an unknown category by a factor of $c$.

2. Category is rapidly identified from unprimed pictures in some (relatively brief) duration, $d_{U c}$.

3. Category identification is a history-independent event. Once a picture's category is known, how it was identified is immaterial, from the perspective of subsequent processing. In particular, a picture whose category has been identified in the absence of a prime is subsequently processed in the same way as a picture whose category has been provided by a prime.

\section{The Model's Account of Our Data}

We show that the model implies that primed and unprimed performance curves are multiplicatively related at short exposure durations but additively related at longer exposure durations. We then estimate unprimed category identification times for Experiments 1-3. Last, we demonstrate that the model correctly predicts the data patterns in Experiments 1-3.

Derivation of horizontal separation between performance curves. The short-duration multiplicative effects and the long-duration additive effects (see Figure 5) are implied by the model for the following reason: According to Assumptions 1 and 2, visual encoding is speeded in a primed condition, in 


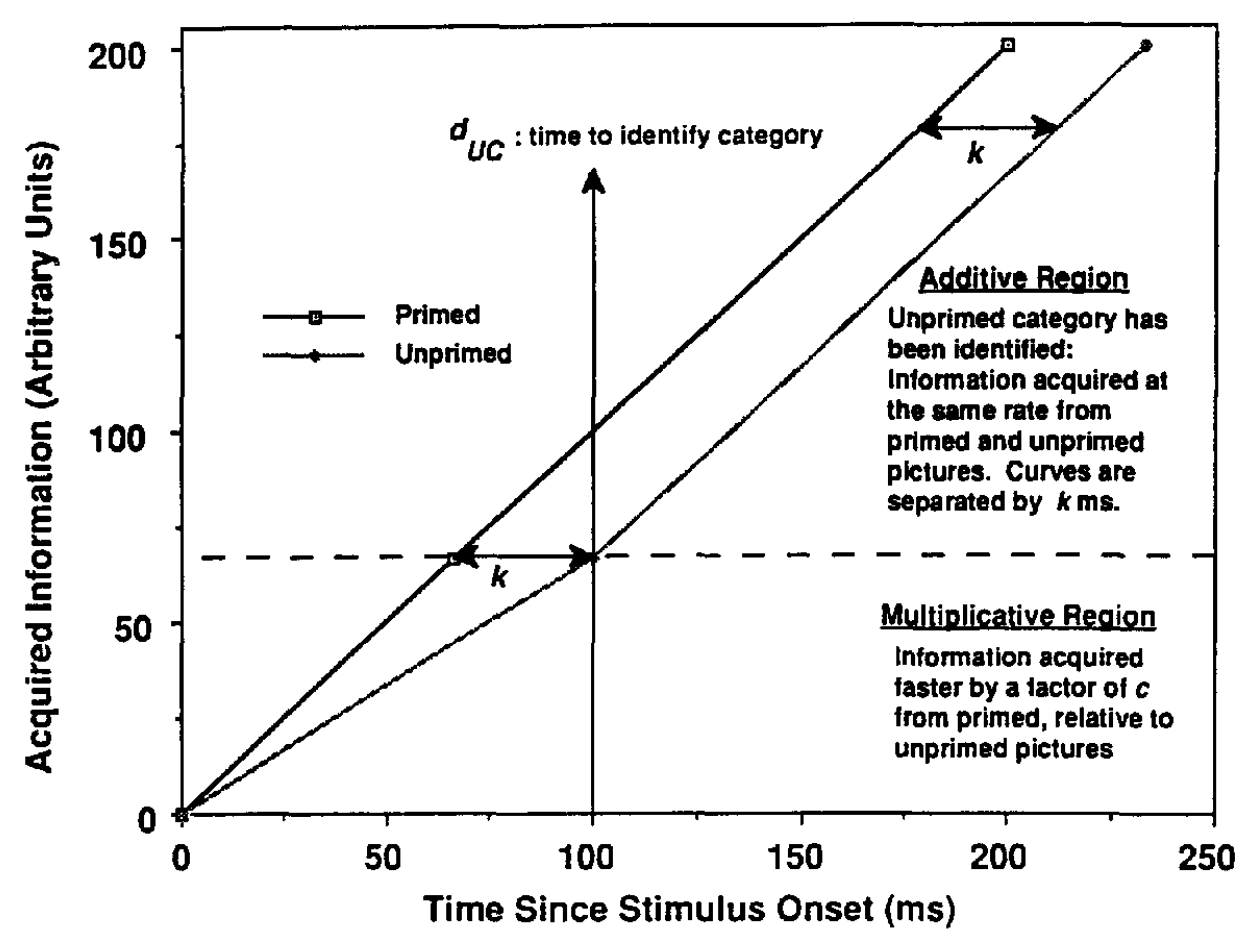

Figure 5. Model predictions about horizontal relations between primed and unprimed performance curves. (In this illustration, $d_{U C}$, the time to identify the category of an unprimed picture, has been set to $100 \mathrm{~ms}$.)

relation to an unprimed condition, up to the point that the category is identified from an unprimed picture. Therefore, at short exposure durations, before unprimed category identification, primed and unprimed performance curves must be described by a multiplicative model and separated horizontally by the constant factor $c$.

Once an unprimed picture's category has been identified, there is no difference between it and a primed picture; both constitute stimuli of known category. According to Assumption 3, subsequent processing is independent of whether the picture had been primed or not; after category identification, processing proceeds in an identical manner for primed and unprimed pictures. Therefore, primed and unprimed performance curves must be separated by a constant horizontal duration, which specifies an additive model.

To carry out the derivations, we let $s$ (in milliseconds) be the horizontal separation between primed and unprimed performance curves:

$$
s=d_{U_{p}}-d_{P_{p}},
$$

where $d_{U_{s}^{\prime}}$ and $d_{P_{p}}$ represent the durations required to achieve performance level $p$ for unprimed and primed pictures. Suppose that an unprimed picture's category is identified at duration $d_{U C} \mathrm{~ms}$ after picture onset. For unprimed exposures shorter than $d_{U c}, d_{P_{p}}=d_{v_{p}} / c$; thus

$$
s=d_{v_{p}}-d_{v_{p}} / c \text {. }
$$

For unprimed durations longer than $d_{U c}, s$ is a constant that, in keeping with past terminology, we call $k$. In summary, therefore,

$$
s=d_{U_{p}}-d_{U_{p}} / c \text {, for } d \leq d_{U C}, \text { and } k \text {, for } d>d_{U C} .
$$

Derivation of $d_{c c}$. As indicated in Figure 5, the maximum horizontal separation between curves, $k$, occurs at the time of unprimed category identification-that is, at duration $d_{v c}$. Accordingly, one can find the value of $d_{U C}$ by substituting $d_{V C}$ for $d_{U_{p}^{\prime}}$ in Equation 3:

$$
k=d_{u c}-d_{u c} / c .
$$

Solving for $d_{v c}$ in Equation 5,

$$
d_{v c}=c k /(c-1) \text {. }
$$

Estimating $c, k$, and $d_{U C}$ for Experiments $1-3$. For each of Experiments 1-3, we estimated $c$ from the relation between primed and unprimed performance curves at short exposure durations and $k$ from the relation between primed and unprimed performance curves at the longest durations. In Table 5 we present these estimates along with the corresponding estimates of $d_{u C}$ found through Equation 6 .

Table 5

Model Parameters Estimated for Experiments 1-3

\begin{tabular}{cccc}
\hline Experiment & $c$ & $k$ (ms) & $d_{U C}(\mathrm{~ms})$ \\
\hline 1 & 1.57 & 37 & 102 \\
2 & 1.49 & 55 & 167 \\
3 & 1.17 & 12 & 83 \\
\hline
\end{tabular}


The estimates of $d_{U C}$ ranged from 83 to $167 \mathrm{~ms}$ over the three experiments. This is in the general range of estimates reported by others (e.g., Biederman et al., 1973; Potter, 1975, 1976).

In Experiments 1 and 2, we were concerned with simple objects. The estimates of $c$, the speedup factor engendered by category knowledge, that we obtained were similar in the two experiments: 1.57 and 1.49. However, the estimates of both $k$ and $d_{v c}$ were lower for Experiment 1 than for Experiment 2. Why is this?

Performance in Experiment 1 required only category identification; when category identification is complete, performance should be perfect. Accordingly, the Experiment 1 priming effect should be described completely by a multiplicative model, as indeed it is. The longest exposure duration used in Experiment 1 (90 ms) was not, by any estimate, sufficient for completion of unprimed category identification (and Experiment 1 performance never reached ceiling). At the longest Experiment 1 exposure durations, the horizontal difference between the primed and unprimed curves was $37 \mathrm{~ms}$, which, perforce, constituted our estimate of $k$. If we had used longer exposure durations, we may well have estimated $k$ to be greater, which would have produced a concomitantly greater estimate of $d_{U c}$ (see Equation 6).

In Experiment 2, in contrast, we measured visual information acquisition independently of category identification. During test, targets and distractors came from the same categories; performance therefore depended on the amount of visual information acquired during study. Furthermore, we used exposure durations that were considerably longer than those in Experiment 1. Accordingly, our estimate of $k$ is not likely to be biased as it was in Experiment 1. For this reason, we have more faith in the $d_{L C}$ and $k$ estimates from Experiment 2 than in those from Experiment 1. However, the estimates of $c$, based as they are on short-exposure-duration data, should be unbiased and accordingly should not differ from one another (except insofar as the actual stimuli were different in the two experiments). Indeed, the two estimates of $c$ are, as noted, quite similar.

Sufficiency of the model: A demonstration. To demonstrate the sufficiency of our model, we have derived predictions for Experiments 1-3. To do so, we add an auxiliary assumption: that the progression of picture processing over time can be characterized by a single number, which, for convenience, we term acquired information. Performance is assumed to be monotonically related to acquired information.

Because we are interested in predicting horizontal relations among performance curves and because, as we have noted earlier, horizontal relations are invariant over monotonic transformations, we are at liberty to scale our units of acquired information any way we wish, as long as acquired information increases over exposure duration. For convenience, we characterize information from a primed picture as being equal to the picture's exposure duration in milliseconds (this characterization also went into the construction of Figure 5). Assumptions 2 and 3 of the model, along with the estimates of $c$ and $k$ (Table 5), then allow us to compute acquired information from unprimed pictures at any given exposure duration and, in particular, at the exposure durations that we used in our experiments.
To assess the fit's adequacy, we constructed scatterplots relating our dependent variables (identification and hit probabilities that we observed from all conditions of Experiments 1-3) to our hypothetical construct (acquired information that the model specifies for all conditions of Experiments 1-3). These scatterplots are shown in Figure 6. The rank-order correlation between the dependent variable and our hypothetical construct is perfect in all cases. This constitutes as good a confirmation of our model as is possible, given the weak monotonicity assumption that links the model to the data. The functions in Figure 6 constitute empirical estimates of the hypothetical monotonic functions that link the model to the data.

\section{Relations of Our Findings to Previous Research}

We have already noted that there is a substantial literature on priming but that very little of this literature is concerned with priming effects on early visual processing. Accordingly, our findings supplement the priming literature in several ways.

\section{What Does Category Knowledge Do?}

Our data indicate that priming a picture with its category name affects perceptual processing of the picture. There clearly was no visual similarity between the primes (alphanumeric strings) and the targets (pictures). Rather, appropriate primes named the targets' categories; the relation was semantic. Our finding that priming affects relatively early perceptual processes (such as those involved in acquiring visual information from stimuli) implies that perceptual and semantic processing systems are highly interconnected. A model that includes such interconnections was proposed by McClelland and Rumelhart (1981; Rumelhart \& McClelland, 1982) to account for context effects in letter perception. We do not claim that their model predicts the specific pattern of multiplicative and additive effects found in our experiments; to determine whether the model could predict such effects would require instantiating it with stronger assumptions, which is beyond the scope of our research. However, McClelland and Rumelhart's model does predict that priming affects perceptual processing, and it provides a relatively straightforward explanation of how semantic and perceptual processes can affect one another. For this reason we briefly describe it.

In their model, McClelland and Rumelhart proposed a hierarchy of increasingly complex levels of visual analysis, including feature detection, letter identification, word identification, and so on. Processing levels are highly interconnected. Each level is represented as a network of linked nodes that gather activation; processing takes the form of activation spread both within and between levels. At any level, a node is activated (identification occurs) when its activation level exceeds some threshold. Top-down and bottom-up processing are both seen as providing activation. Prior category presentation can spread top-down activation to nodes representing category-relevant visual features. The result is that less information is required from the physical stimulus in order to detect primed features; they will therefore be detected more rapidly than will unprimed features. According to this expla- 

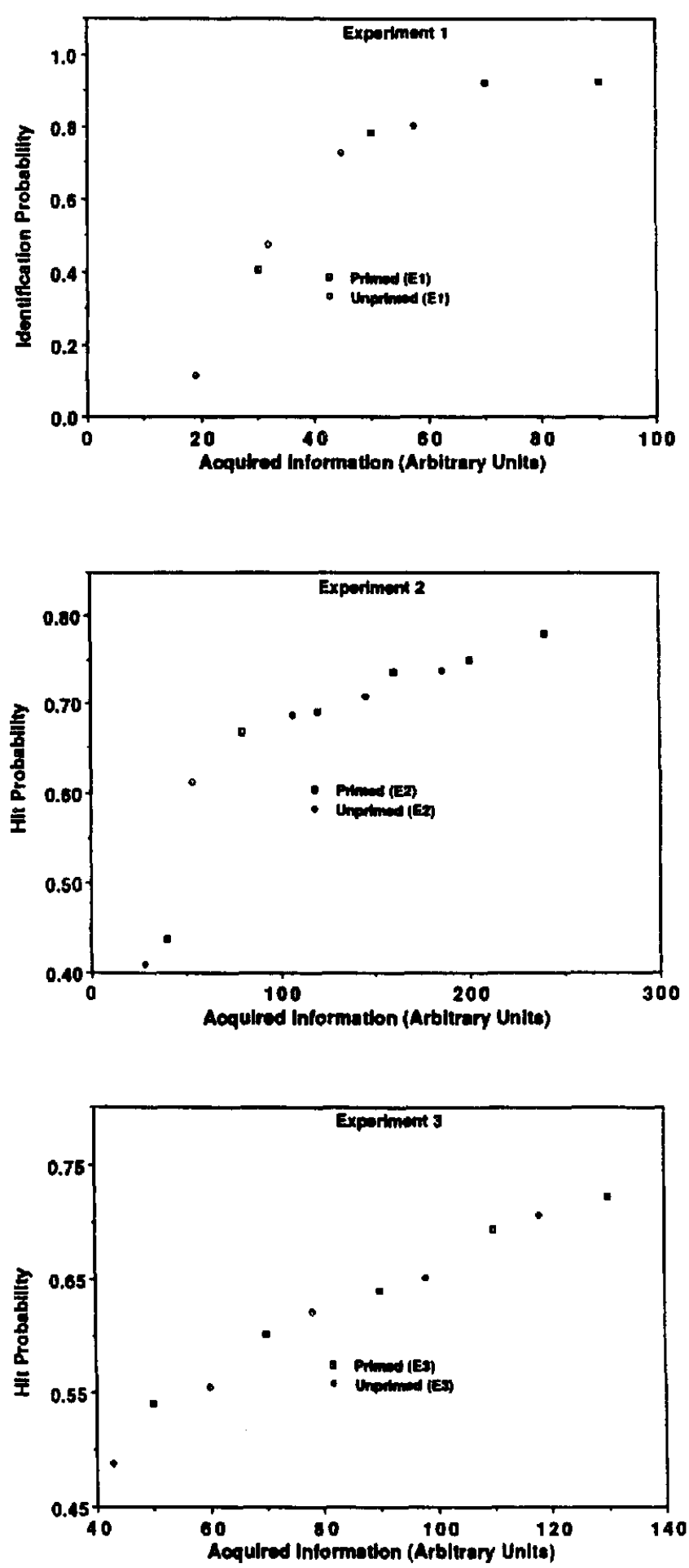

Figure 6. Scatterplots relating obtained performance to theoretically derived "acquired information" for Experiments 1, 2, and 3 (top, middle and bottom panels).

nation, the time to detect any feature is reduced by a constant amount when that feature is primed. The model therefore is additive at the level of individual features. However, because performance depends on the acquisition of many features, the model can produce the multiplicative effect that we propose and that is found in Experiments 1-3.

According to this explanation, category activation spreads to category-relevant feature detectors. Initially, features in primed pictures have an activation source that is not present in unprimed pictures. However, once the node representing an unprimed picture's category becomes active (at time $d_{v C}$ ), there is no longer any advantage for primed pictures because there is now equivalent top-down activation. We are not the first authors to claim that primes facilitate picture perception by directly affecting feature detectors (cf. C. Warren \& Morton, 1982).

\section{Semantic Priming Versus Repetition Priming}

In our experiments, primes were always categories; in a sense the priming effects that we obtained therefore represent examples of repetition priming. In many priming experiments, semantically related items, rather than categories, serve as primes. McClelland and Rumelhart's (1981) model predicts perceptual effects of semantically related primes as follows: At some time after prime onset, the node at the semantic level representing the prime becomes active. Activation spreads from that node to nodes representing prime-relevant concepts (one of which represents the target in an AP condition). Top-down activation then spreads from those nodes to their representations at the featural level. For objects, then, the effect of priming with a semantically related item should be the same as priming by the object name because both types of primes serve to decrease the time needed to acquire targetrelevant features. However, less top-down activation would be expected to spread to the target's feature set after a semantically related prime. The increase in encoding rate, $c$, should thus be less than that provided by category primes.

\section{Levels of Priming Effects}

Priming has been shown to affect various stages of cognitive processing. We have shown that one of these stages is perceptual: It is initial information acquisition from a visual stimulus. In order to fully understand priming effects, it is necessary to identify specific effects of primes with the specific processing level at which the effect occurs.

As an example, consider that in all four of our experiments there was no cost associated with unrelated primes. Many experimenters, using timing parameters similar to ours, have reported such cost (cf. Neely, 1977; Posner \& Snyder, 1975). Cost in an unrelated-prime condition is generally associated with controlled processing and usually is attributed to postperceptual processes, such as response selection. Often, cost occurs in an unrelated-prime condition when primes produce a response bias or criterion shift rather than a change in sensitivity. Response selection explanations for our results were ruled out by Experiments 2-4. In our experiments performance was determined by perceptual processing. Visual feature acquisition is probably outside the direct control of subjects. Because the processing that determined performance in our experiments probably cannot be controlled, and because cost is associated with controlled processing, it is not 
surprising that we failed to observe any cost associated with unrelated primes.

\section{Conclusions}

We have demonstrated that priming directly affects the acquisition of visual information from pictures. The priming effect that we have found can be readily explained if it is assumed that (a) knowledge of a picture's category increases the rate at which visual information is encoded and (b) once a picture's category is known, subsequent processing is independent of the means by which the category was identified.

The postulation of additive and multiplicative models was instrumental in our arriving at these conclusions. We believe that this methodological technology will prove useful in unveiling other picture-processing stages. Research in quest of this goal is presently in progress.

\section{References}

Anderson, J. R. (1974). Retrieval of propositional information from long-term memory. Cognitive Psychology, 6, 451-474.

Biederman, I., Glass, A., \& Stacy, E. (1973). Searching for objects in real-world scenes. Journal of Experimental Psychology, 97, 22-27.

Burke, D. M., White, H., \& Diaz, D. L. (1987). Semantic priming in younger and older adults: Evidence for age constancy in automatic and attentional processes. Journal of Experimental Psychology: Human Perception and Performance, 13, 79-88.

Carr, T. H., McCauley, C., Sperber, R. D., \& Parmalee, C. M. (1982). Words, pictures, and priming: On semantic activation, conscious identification, and automaticity of information processing. Journal of Experimental Psychology: Human Learning and Memory, 8, 757-777.

Dosher, B. A. (1976). The retrieval of sentences from memory: A speed-accuracy study. Cognitive Psychology, 8, 291-310.

Dosher, B. A. (1984). Discriminating preexperimental (semantic) from learned (episodic) associations: A speed-accuracy study. Cognitive Psychology, 16, 519-555.

Durso, F. T., \& Johnson, M. K. (1979). Facilitation in naming and categorizing repeated pictures and words. Journal of Experimental Psychology: Human Learning and Memory, 5, 449-459.

Intraub, H. (1981). Identification and processing of briefly-glimpsed visual scenes. In D. E. Fisher, R. A. Monty, \& J. W. Senders (Eds.), Eye Movements: Cognition and visual perception (pp. 181-190). Hillsdale, NJ: Erlbaum.

Irwin, D. I., \& Lupker, S. J. (1983). Semantic priming of pictures and words: A levels of processing approach. Joumal of Verbal Learning and Verbal Behavior, 22, 45-60.

Kroll, J. F., \& Curley, J. (1986, November). Conceptual facilitation in bilingual word recognition. Paper presented at the TwentySeventh Annual Meeting of the Psychonomic Society, New Orleans.

Kroll, J. F, \& Potter, M. C. (1977, November). Can pictures and words prime each other? Paper presented at the Eighteenth Annual Meeting of the Psychonomic Society, Washington, DC.

Kroll, J. F., \& Potter, M. C. (1984). Recognizing words, pictures, and concepts: A comparison of lexical, object, and reality decisions. Journal of Verbal Learning and Verbal Behavior, 23, 39-66.

Loftus, G. R. (1978). On interpretation of interactions. Memory \& Cognition, 6, 312-319.
Loftus, G. R. (1982). Eye fixations on text and scenes. In K. Rayner (Ed.), Eye movements in reading: Perceptual and language processes (chap. 21). New York: Academic Press.

Loftus, G. R., Gillispie, S., Tigre, R. A., \& Nelson, W. W. (1984). An Apple Il-based slide projector laboratory. Behavior Research Methods, Instruments, and Computers, 16, 447-453.

Loftus, G. R., Hanna, A., \& Lester, L. (1988). Conceptual masking: How one picture steals attention from another picture. Cognitive Psychology, 20, 237-282.

Loftus, G. R., \& Hogden, J. (1988). Picture perception: Information extraction and phenomenological appearance. In G. H. Bower (Ed.), The psychology of learning and motivation (Vol. 22, pp. 139191). New York: Academic Press.

Loftus, G. R., \& Mackworth, N. H. (1978). Cognitive determinants of fixation location during picture viewing. Journal of Experimental Psychology: Human Perception and Performance, 4, 565-572.

Loftus, G. R., Nelson, W. W., \& Kallman, H. J. (1983). Differential acquisition rates for different types of information from pictures. Quarterly Journal of Experimental Psychology, 35, 187-198.

Lupker, S. J. (1979). The semantic nature of response competition in the picture-word interference task. Memory \& Cognition, 7, 485495.

Lupker, S. J., \& Katz, A. N. (1982). Can automatic picture processing influence word judgments? Journal of Experimental Psychology: Learning, Memory, and Cognition, 8, 418-434.

McClelland, J. L., \& Rumelhart, D. E. (1981). An interactive model of context effects in letter perception: Part 1. An account of basic findings. Psychological Review, 88, 375-407.

McKoon, G., \& Ratcliff, R. (1980). Priming in item recognition: The organization of propositions in memory for text. Journal of Verbal Learning and Verbal Behavior, 19, 369-386.

Meyer, D. E., Schvaneveldt, R. W., \& Ruddy, M. G. (1975). Loci of contextual effects on visual word recognition. In P. M. A. Rabbit \& S. Dornic (Eds.), Attention and performance $V$ (pp. 98-118). London: Academic Press.

Morton, J. (1964). The effects of context on the visual duration threshold for words. British Journal of Psychology, 55, 165-180.

Morton, J. (1969). The interaction of information in word recognition. Psychological Review, 76, 165-178.

Neely, J. H. (1977). Semantic priming and retrieval from lexical memory: Roles of inhibitionless spreading activation and limitedcapacity attention. Journal of Experimental Psychology: General, 106, 226-254.

Neely, J. H., \& Durgunoglu, A. (1985). Dissociative episodic and semantic priming effects in episodic and semantic memory. Journal of Memory and Language, 24, 466-489.

Neely, J. H., Schmidt, S. R., \& Roediger, H. L. (1983). Inhibition from related primes in recognition memory. Journal of Experimental Psychology: Learning, Memory, and Cognition, 9, 196-211.

Palmer, S. E. (1975). The effects of contextual scenes on the identification of objects. Memory \& Cognition, 3, 519-526.

Posner, M., \& Snyder, C. R. R. (1975). Facilitation and inhibition in the processing of signals. In P. M. A. Rabbit \& S. Dornic (Eds.), Attention and performance V (pp. 669-682). New York: Academic Press.

Potter, M. C. (1975). Meaning in visual search. Science, 187, 965966.

Potter, M. C. (1976). Short-term conceptual memory for pictures. Journal of Experimental Psychology: Human Learning and Memory, 2, 509-522.

Potter, M. C. (1979). Mundane symbolism: The relations among objects, names, and ideas. In N. Smith \& N. Franklin (Eds.), Symbolic functioning in childhood (pp. 41-65). Hillsdale, NJ: Erlbaum. 
Potter, M. C., \& Faulconer, B. A. (1975). Time to understand pictures and words. Nature, $253,437-438$.

Ratcliff, R., Hockley, W., \& McKoon, G. (1986). Components of activation: Repetition and priming effects in lexical decision and recognition. Journal of Experimental Psychology: General, 114, $435-450$.

Ratcliff, R., \& McKoon, G. (1978). Priming in item recognition: Evidence for the propositional structure of sentences. Journal of Verbal Learning and Verbal Behavior, 17, 403-417.

Ratcliff, R., \& McKoon, G. (1988). A retrieval theory of priming in memory. Psychological Review, 95, 385-408.

Rumelhart, D. E., \& McClelland, J. L. (1982). An interactive activation model of context effects in letter perception: Part 2. The contextual enhancement effect and some tests and extensions of the model. Psychological Review, 89, 60-94.

Seymour, P. H. K. (1973). A model for reading, naming, and comparison. British Journal of Psychology, 64, 35-49.

Smith, M. C. (1986, November). Between-language priming depends on how the prime is processed. Paper presented at the TwentySeventh Annual Meeting of the Psychonomic Society, New Orleans.

Sperber, R. D., McCauley, C., Ragain, R. D., \& Weil, C. M. (1979).
Semantic priming effects on picture and word processing. Memory \& Cognition, 7, 339-345.

Stoddard, P., \& Loftus, G. R. (1988). An IBM-XT compatible, computer-based slide-projector laboratory. Behavior Research Method, Instrumentation, and Computers, 20, 541-551.

Tulving, E., Schacter, D. L., \& Stark, H. A. (1982). Priming effects in word-fragment completion are independent of recognition memory. Journal of Experimental Psychology: Learning, Memory, and Cognition, 8, 336-341.

Warren, C., \& Morton, J. (1982). The effects of priming on picture recognition. British Journal of Psychology, 73, 117-129.

Warren, R. E. (1977). Time and the spread of activation in memory. Journal of Experimental Psychology: Human Learning and Memory, 3, 458-466.

Warrington, E. K., \& Weiskrantz, L. (1968). A new method of testing long-term retention with special reference to amnesic patients. Nature, 217, 972-974.

Received June 28, 1988

Revision received February 9, 1989

Accepted February 9, 1989 